\title{
Complete Set of CHC Tetrahedrons
}

\author{
Pranab Kalita \\ Gauhati University \\ Department of Mathematics \\ Guwahati-781014, Assam, India
}

\author{
Bichitra Kalita \\ Assam Engineering College \\ Department of Computer Applications (M.C.A) \\ Guwahati-781013, Assam, India
}

\begin{abstract}
In this article, using K. W. Roeder's Theorem, some properties of CHC (compact hyperbolic coxeter) tetrahedrons have been developed which are facilitated by the link of graph theory and combinatorics, and it has been found that there are exactly $3 \mathrm{CHC}$ tetrahedrons upto symmetry.
\end{abstract}

\section{General Terms}

Three Dimensional Polytope, Hyperbolic Space, Roland K. W. Roeder Theorem.

\section{Keywords}

Dihedral angles, Face angles, Planar graph, Coxeter.

\section{INTRODUCTION}

The angle between two faces of a polytope, measured from perpendiculars to the edge created by the intersection of the planes is called a dihedral angle. A simple polytope $P$ in $n$ dimensional hyperbolic space $H^{n}$ is said to be coxeter, if the dihedral angles of $P$ are of the form $\frac{\pi}{n}$ where, $n$ is a positive integer $\geq 2$. P. Kalita and B. Kalita [1] found that there are exactly one, four and thirty coxeter Andreev's tetrahedrons having respectively two edges of order $n \geq 6$, one edge of order $n \geq 6$ and no edge of order $n \geq 6, n \in \square$ upto symmetry. There is no complete classification of hyperbolic coxeter polytopes. Vinberg proved in [22] that there are no compact hyperbolic coxeter polytopes in $H^{n}$ when $n \geq 30$. Roland K. W. Roeder's Theorem [10] provides the classification of compact hyperbolic tetrahedron by restricting to non-obtuse dihedral angles. In this article, using K. W. Roeder's Theorem, some properties of CHC (compact hyperbolic coxeter) tetrahedrons have been developed which are facilitated by the link of graph theory and combinatorics, and it has been found that there are exactly $3 \mathrm{CHC}$ tetrahedrons upto symmetry.

The paper is organised as follows:

The section 1 includes introduction. The section 2 includes some basic terminologies from graph theory and geometry. The section 3 focuses some already existed results on graph theory and geometry. Main results and conclusions are included in the sections 4 and 5 respectively.

\section{BASIC TERMINOLOGIES}

Definition 2.1: Let $P$ be a polyhedron. The abstract graph of $P \quad$ is denoted by $G(P)$ and is defined as $G(P)=(V(P), E(P))$, where $V(P)$ is the set of vertices of
$P$ and two vertices $x, y \in V(P)$ are adjacent if and only if $(x, y)$ is an edge of $P$.

Definition 2.2: If the dihedral angle of an edge of a compact hyperbolic polytope is $\frac{\pi}{n}, n$ is a positive number, then $n$ is said to be the order of the edge. A trivalent vertex is defined to be of order $(l, m, n)$ if the three edges at that vertex are of order $l, m, n$.

Definition 2.3: A compact polytope in hyperbolic space with coxeter dihedral angles is called a CHC (compact hyperbolic coxeter) polytope.

Suppose the dihedral angles at the edges $e_{1}, e_{2}, e_{3}, e_{4}, e_{5}, e_{6}$ of a coxeter tetrahedron are respectively $\frac{\pi}{n_{1}}, \frac{\pi}{n_{2}}, \frac{\pi}{n_{3}}, \frac{\pi}{n_{4}}, \frac{\pi}{n_{5}}, \frac{\pi}{n_{6}}$ as shown in figure 1.

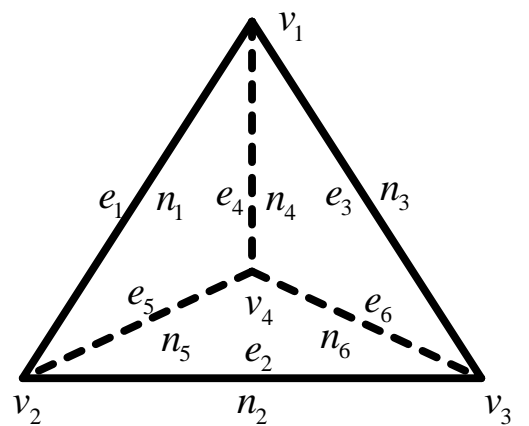

Figure 1: CHCT $=\left[\boldsymbol{n}_{1}, \boldsymbol{n}_{2}, \boldsymbol{n}_{3}, \boldsymbol{n}_{4}, \boldsymbol{n}_{5}, \boldsymbol{n}_{6}\right]$.

Then the CHC tetrahedron is denoted as

$$
\text { CHCT }=\left[n_{1}, n_{2}, n_{3}, n_{4}, n_{5}, n_{6}\right] .
$$

Definition 2.4: A cell complex $C$ on $S^{2}$ is called trivalent if each vertex is the intersection of three faces.

Definition 2.5: A 3-dimensional combinatorial polytope is a cell complex $C$ on $S^{2}$ that satisfies the following conditions:

(a) Each edge of $C$ is the intersection of exactly two faces

(b) A nonempty intersection of two faces is either an edge or a vertex.

(c) Each face is enclosed by not less than 3 edges.

Any trivalent cell complex $C$ on $S^{2}$ that satisfies the above three conditions is said to be abstract polyhedron.

In this work, the main concentration is on 3-dimensional compact hyperbolic polytopes whose base spaces are 
homeomorphic to a convex polyhedron and whose sides are silvered. The compact hyperbolic polyhedron is simple, therefore, the combinatorial polyhedron of a compact hyperbolic polyhedron can be known from 3-connected planar graph of the polyhedron. In case of compact hyperbolic tetrahedron, the corresponding 3-connected planar graph has 4 vertices, 6 edges, 4 faces (excluding exterior one).

Hyperbolic geometry is difficult to visualize as many of its theorems are contradictory to similar theorems of Euclidean geometry which are very similar to us. Therefore technology has been used in creation of geometric models in Euclidean space to visualize Hyperbolic geometry. Some developed models are The Klein Model, The Poincare Model etc. Hyperbolic [10] planes in these models correspond to Euclidean hemispheres and Euclidean planes that intersect the boundary perpendicularly. Furthermore, these models are correct conformally. That is, the hyperbolic angle between a pair of such intersecting hyperbolic planes is exactly the Euclidean angle between the corresponding spares or planes.

\section{KNOWN RESULTS}

Theorem 3.1: (Blind and Mani) If $P$ is a convex polyhedron, then the graph $G(P)$ determines the entire combinatorial structure of $P$. In other words, if two simple polyhedral have isomorphic graphs, then their combinatorial polyhedral are also isomorphic.

Theorem 3.2: (Ernst Steinitz) A graph $G(P)$ is a graph of a 3-dimensional polytope $P$ if and only if it is simple, planar and 3-connected.

Lemma 3.3: (E. M. Andreev [23]) Suppose that three planes $P_{v_{1}}, P_{v_{2}}, P_{v_{3}}$ intersect pair-wise in $H^{3}$ with non-obtuse dihedral angles $\alpha_{i}, \alpha_{j}, \alpha_{k}$. Then, $P_{v_{1}}, P_{v_{2}}, P_{v_{3}}$ intersect at a vertex in $\overline{H^{3}}$ if and only if $\alpha_{i}+\alpha_{j}+\alpha_{k} \geq \pi$. The planes intersect in $H^{3}$ if and only if the inequality is strict (that is $\left.\alpha_{i}+\alpha_{j}+\alpha_{k}>\pi\right)$

Lemma 3.4: (E. M. Andreev [23]) Given a trivalent vertex of a hyperbolic polyhedron, we can compute the angles of the faces in terms of the dihedral angles. If the dihedral angles are non-obtuse, these angles are also $\leq \frac{\pi}{2}$.

Proof: Let $v$ be a finite trivalent vertex of a polyhedron $P$. After an appropriate isometry, we can assume that $v$ is the origin in the Poincare ball model, so that the faces at $v$ are subsets of Euclidean planes through the origin. A small sphere centered at the origin will intersect $P$ in a spherical triangle $Q$ whose angles are the dihedral angles between faces. Call these angles $\alpha_{1}, \alpha_{2}, \alpha_{3}$.

The edge lengths of $Q$ are precisely the angles in the faces at the origin. Suppose that $Q$ has edge length $\left(\beta_{1}, \beta_{2}, \beta_{3}\right)$ with the edge $\beta_{i}$ opposite the angle $\alpha_{i}$ for each $i=1,2,3$, the law of cosines in spherical geometry states that:

$$
\cos \left(\beta_{i}\right)=\frac{\cos \left(\alpha_{i}\right)+\cos \left(\alpha_{j}\right) \cos \left(\alpha_{k}\right)}{\sin \left(\alpha_{j}\right) \sin \left(\alpha_{k}\right)}
$$

Hence the face angles are calculated from the dihedral angles. They are non-obtuse, since the right-hand side of the equation is positive for $\alpha_{i}, \alpha_{j}, \alpha_{k}$ are non-obtuse.

Theorem 3.5: (Roland K. W. Roeder, 2013, [10]) Let $\alpha_{1}, \cdots, \alpha_{6}$ be a set of proposed non-obtuse dihedral angles and let $\beta_{1}\left(\alpha_{1}, \cdots, \alpha_{6}\right), \cdots, \beta_{12}\left(\alpha_{1}, \cdots, \alpha_{6}\right)$ be the face angles given by equation (1), corresponding to these proposed dihedral angles.

There is a compact hyperbolic tetrahedron with dihedral angles $\alpha_{1}, \cdots, \alpha_{6}$ if and only if

(1) For each edge $e_{i}, 0<\alpha_{i} \leq \frac{\pi}{2}$.

(2) Whenever 3 distinct edges $e_{i}, e_{j}, e_{k}$ meet at a vertex, $\alpha_{i}+\alpha_{j}+\alpha_{k}>\pi$.

(3) For each face the sum of the face angles satisfies $\beta_{i}+\beta_{j}+\beta_{k}<\pi$.

Furthermore this tetrahedron is unique.

In this article, the dihedral angles of a tetrahedron are restricted to be coxeter and hence non-obtuse as well. Therefore, the concentration is only on the CHC tetrahedrons given by the Theorem 3.5

\section{MAIN RESULTS}

Now the main results will be established below.

Theorem 4.1: In a CHC tetrahedron $T$, there is no vertex of order of the form $(2,2, n \geq 2)$.

Proof: Suppose there exists at least one vertex of order $(2,2, n \geq 2)$. Therefore the dihedral angles are $\left(\alpha_{i}, \alpha_{j}, \alpha_{k}\right)=\left(\frac{\pi}{2}, \frac{\pi}{2}, \frac{\pi}{n} ; n \geq 2\right)$. The corresponding face angles are calculated by using the equation (1):

$$
\begin{aligned}
\cos \left(\beta_{i}\right) & =\frac{\cos \left(\alpha_{i}\right)+\cos \left(\alpha_{j}\right) \cos \left(\alpha_{k}\right)}{\sin \left(\alpha_{j}\right) \sin \left(\alpha_{k}\right)} \\
& =\frac{\cos \frac{\pi}{2}+\cos \frac{\pi}{2} \cos \frac{\pi}{n}}{\sin \frac{\pi}{2} \sin \frac{\pi}{n}} \\
& =0
\end{aligned}
$$

By lemma 3.4, $0<\beta_{i} \leq \frac{\pi}{2}$, therefore $\beta_{i}=\frac{\pi}{2}$. Similarly it can be deduced that $\beta_{j}=\frac{\pi}{2}$ and $\beta_{k}=\frac{\pi}{n}$. Now,

$$
\beta_{i}+\beta_{j}+\beta_{k}=\frac{\pi}{2}+\frac{\pi}{2}+\frac{\pi}{n}=\pi+\frac{\pi}{n} \nless \pi
$$

That is the Roeder's $3^{\text {rd }}$ condition (of Theorem 3.5) is not satisfied. Hence, in a CHC tetrahedron $T$, there is no vertex of order of the form $(2,2, n \geq 2)$.

Theorem 4.2: In a CHC tetrahedron $T$, there is no vertex of order of the form $(2,3,3)$. 
Proof: Suppose there exists at least one vertex of order $(2,3,3)$. Therefore the dihedral angles are $\left(\alpha_{i}, \alpha_{j}, \alpha_{k}\right)=\left(\frac{\pi}{2}, \frac{\pi}{3}, \frac{\pi}{3}\right)$ and the face angles given by (1) are:

$$
\left(\beta,_{i} \beta_{j}, \beta_{k}\right)=\left(\cos ^{-1}\left(\frac{1}{3}\right), \cos ^{-1}\left(\frac{1}{\sqrt{3}}\right), \cos ^{-1}\left(\frac{1}{\sqrt{3}}\right)\right)
$$

But $\beta_{i}+\beta_{j}+\beta_{k} \nless \pi$, That is the Roeder's $3^{\text {rd }}$ condition (of Theorem 3.5) is not satisfied. Hence, in a CHC tetrahedron $T$ , there is no vertex of order of the form $(2,3,3)$.

Theorem 4.3: In a CHC tetrahedron $T$, the order of each of the vertices is one of the forms: $(2,3,4),(2,3,5)$.

Proof: Let $e_{i}, e_{j}, e_{k}$ be three distinct edges of orders $n_{i}, n_{j}, n_{k}$ meet at one vertex $v$ in $T$. The respective dihedral angles of $e_{i}, e_{j}, e_{k}$ at $v$ are $\frac{\pi}{n_{i}}, \frac{\pi}{n_{j}}, \frac{\pi}{n_{k}}$, with positive integers $n_{i}, n_{j}, n_{k} \geq 2$. By Roeder's $2^{\text {nd }}$ condition (of Theorem 3.5):

$$
\alpha_{i}+\alpha_{j}+\alpha_{k}>\pi \Rightarrow \frac{\pi}{n_{i}}+\frac{\pi}{n_{j}}+\frac{\pi}{n_{k}}>\pi \Rightarrow \frac{1}{n_{i}}+\frac{1}{n_{j}}+\frac{1}{n_{k}}>1
$$

So, upto permutations, the triples $\left(n_{i}, n_{j}, n_{k}\right)$ are respectively $(2,2, n \geq 2),(2,3,3),(2,3,4),(2,3,5)$. The vertices of order $(2,2, n \geq 2)$ are not possible by Theorem 4.1 and the vertices of order $(2,3,3)$ are not possible by Theorem 4.2. For the vertices of order $\left(n_{i}, n_{j}, n_{k}\right)=(2,3,4) \quad$ and $\left(n_{i}, n_{j}, n_{k}\right)=(2,3,5)$; the corresponding dihedral angles are:

$$
\left(\alpha_{i}, \alpha_{j}, \alpha_{k}\right)=\left(\frac{\pi}{2}, \frac{\pi}{3}, \frac{\pi}{4}\right),\left(\alpha_{i}, \alpha_{j}, \alpha_{k}\right)=\left(\frac{\pi}{2}, \frac{\pi}{3}, \frac{\pi}{5}\right)
$$

For $\left(\alpha_{i}, \alpha_{j}, \alpha_{k}\right)=\left(\frac{\pi}{2}, \frac{\pi}{3}, \frac{\pi}{4}\right)$, the face angles given by (1) are:

$$
\left(\beta,{ }_{i} \beta_{j}, \beta_{k}\right)=\left(\cos ^{-1}\left(\frac{1}{\sqrt{3}}\right), \frac{\pi}{4}, \cos ^{-1}\left(\sqrt{\frac{2}{3}}\right)\right)
$$

And $\beta_{i}+\beta_{j}+\beta_{k}<\pi$, That is the Roeder's $3^{\text {rd }}$ condition (of Theorem 3.5) is satisfied. Hence, in a CHC tetrahedron $T$, there is vertex of order of the form $(2,3,4)$.

For $\left(\alpha_{i}, \alpha_{j}, \alpha_{k}\right)=\left(\frac{\pi}{2}, \frac{\pi}{3}, \frac{\pi}{5}\right)$, the face angles given by (1) are: $\left(\beta,{ }_{i} \beta_{j}, \beta_{k}\right)=\left(\cos ^{-1}(0.7947), \cos ^{-1}(0.8506295), \cos ^{-1}(0.93415)\right)$ And $\beta_{i}+\beta_{j}+\beta_{k}<\pi$, That is the Roeder's $3^{\text {rd }}$ condition (of Theorem 3.5) is satisfied. Hence, in a CHC tetrahedron $T$, there is vertex of order of the form $(2,3,5)$. Therefore in a CHC tetrahedron $T$, the order of the edges at one vertex is one of the forms: $(2,3,4),(2,3,5)$.

Corollary 4.4: In a CHC tetrahedron $T$, the number of edges of order 2 at one vertex is exactly one.

Proof: In a CHC tetrahedron $T$, the order of the edges at one vertex is one of the forms: $(2,3,4),(2,3,5)$. Therefore the number of edges of order 2 at one vertex is exactly one.
Corollary 4.5: In a CHC tetrahedron $T$, the edges of order 2 must be disjoint.

Proof: Suppose there exists at least two adjacent edges of order 2 . Then the order of the vertex at which the two edges of order 2 are adjacent becomes $(2,2, n \geq 2)$. This is not possible by Theorem 4.1. Therefore the edges of order 2 must be disjoint.

The following Theorem 4.6 and corollary 4.7 have been taken from [1] which will be used in this present work.

Theorem 4.6: In a tetrahedron $T$, if any three vertices are of same order, then the fourth vertex is also of same order.

Corollary 4.7: In a tetrahedron $T$, the number of same order vertices can be either 2 or 4.

Theorem 4.8: Let $T$ be a CHC tetrahedron with at least one vertex is of order $(2,3,4)$. Then there are exactly 2 such $T$ upto symmetry.

Proof: Let $T$ be a CHC tetrahedron with at least one vertex is of order $(2,3,4)$. By corollary 4.7 , in a $\mathrm{CHC}$ tetrahedron

$T$, the number of same order vertices can be either 2 or 4 . Therefore, there will be three cases: Case 1: all (four) the vertices are of order $(2,3,4)$, Case 2: two vertices are of order $(2,3,4)$ and Case 3: one vertex is of order $(2,3,4)$.

Case 1: All the vertices of $T$ are of order $(2,3,4)$.

In this case, there will be exactly $1 T$ of this type as shown in figure 2 upto symmetry.

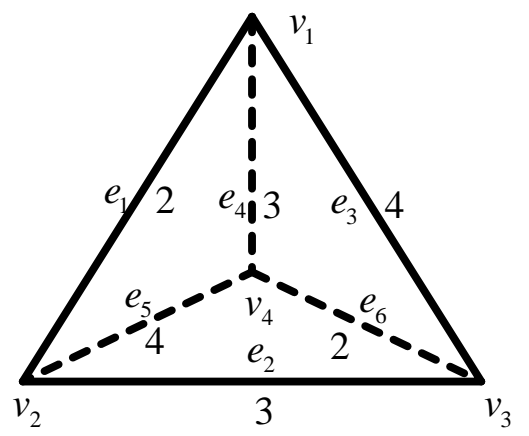

Figure 2: CHCT-1 $=[2,3,4,3,4,2]$

Case 2: Two vertices are of order $(2,3,4)$.

Obviously the two vertices of order $(2,3,4)$ with either disjoint edges of order 2 or common edge of order 2 .

Case 2.1: Two vertices of order $(2,3,4)$ with disjoint edges of order 2.

Suppose the vertices $v_{1}$ and $v_{3}$ are of order $(2,3,4)$ with disjoint edges $e_{1}$ and $e_{6}$ of order 2 . 


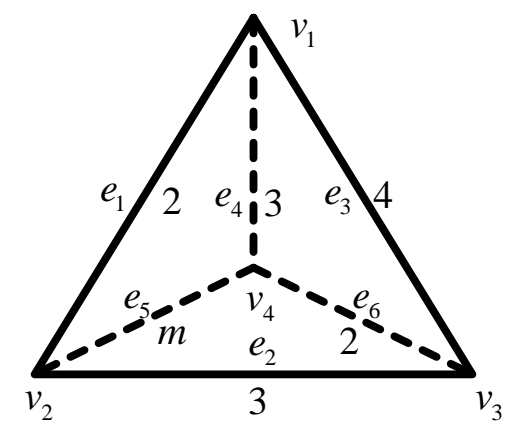

Figure 3: CHC Tetrahedron with Two vertices of order $(2,3,4)$ having disjoint edges of order 2.

Then the possibilities of orders for $e_{5}$ are $m=2,3,4,5$. For $m=2$, the order of $v_{2}$ becomes $(2,2,3)$ which is not possible by Theorem 4.1. For $m=3$, the order of $v_{2}$ becomes $(2,3,3)$ which is not possible by Theorem 4.2. For $m=4$, it falls in case 1 . For $m=5$, there is exactly 1 such $T$ of this type as shown in figure 4 upto symmetry.

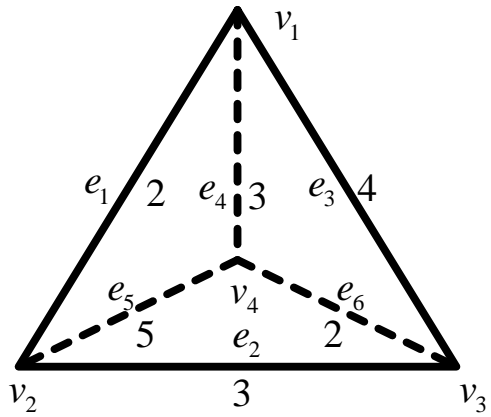

Figure 4: CHCT-2 $=[2,3,4,3,5,2]$

Case 2.2: Two vertices of order $(2,3,4)$ with common edge of order 2 .

Suppose the vertices $v_{1}$ and $v_{2}$ are of order $(2,3,4)$ with common edge $e_{1}$ of order 2 . Then the possibilities are as shown in figures 5 and 6 .

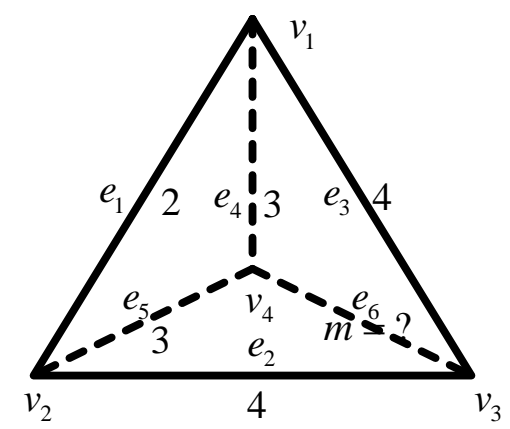

Figure 5: CHC Tetrahedron with Two vertices of order $(2,3,4)$ having common edge of order 2

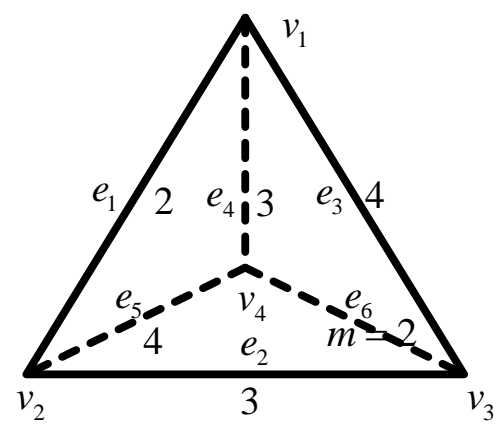

Figure 6: Another CHC Tetrahedron with Two vertices of order $(2,3,4)$ having common edge of order 2 .

In figure 5 , there is no choice for $m$. In figure 6 , only choice is $m=2$ which falls in case 1 .

Case 3: One vertex $v_{1}$ is of order $(2,3,4)$.

By corollary 4.4 , the number of edges of order 2 at one vertex is exactly one and by corollary 4.5, the edges of order 2 must be disjoint. Therefore if $v_{1}$ is of order $(2,3,4)$ with $e_{1}$ is of order 2 , then $e_{6}$ must be of order 2. Suppose the order of the edges $e_{2}$ and $e_{5}$ are $m_{1}$ and $m_{2}$ respectively.

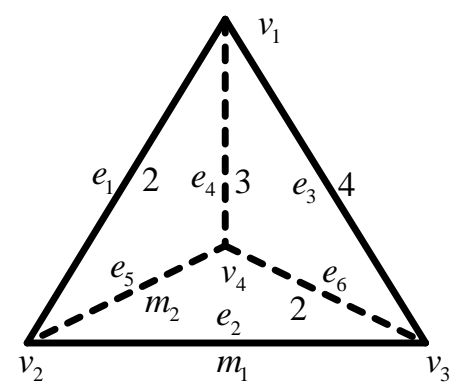

Figure 7: CHC Tetrahedron with one vertex of order $(2,3,4)$

To avoid symmetry, assume $m_{1} \leq m_{2}$. Therefore, $m_{1}=3$ and $m_{2}=3,4,5$ upto symmetry. For $m_{1}=3$ and $m_{2}=3$, the order of $v_{2}$ becomes $(2,3,3)$, which is not possible by Theorem 4.2 and $m_{1}=3, m_{2}=4$ as well as $m_{1}=3, m_{2}=5$ lead to case 2 . Hence, there is no such $T$ of this type.

Theorem 4.9: Let $T$ be a CHC tetrahedron with at least one vertex is of order $(2,3,5)$ and no vertex is of order of the forms $(2,3,4)$. Then there are exactly 1 such $T$ upto symmetry.

Proof: Let $T$ be a CHC tetrahedron with at least one vertex is of order $(2,3,5)$ and no vertex is of order of the forms $(2,3,4)$. By corollary 4.7 , in a CHC tetrahedron $T$, the number of same order vertices can be either 2 or 4 . Therefore, there will be three cases: Case 1: all (four) the vertices are of order $(2,3,5)$, Case 2 : two vertices are of order $(2,3,5)$ and Case 3: one vertex is of order $(2,3,5)$.

Case 1: All the vertices of $T$ are of order $(2,3,5)$.

In this case, there is exactly $1 T$ as shown in figure 8 upto symmetry. 


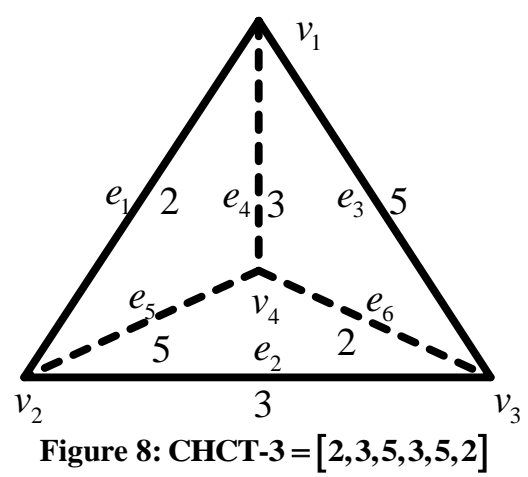

Case 2: Two vertices are of order $(2,3,5)$.

Obviously the two vertices of order $(2,3,5)$ with either disjoint edges of order 2 or common edge of order 2

Case 2.1: Two vertices of order $(2,3,5)$ with disjoint edges of order 2.

Suppose the vertices $v_{1}$ and $v_{3}$ are of order $(2,3,5)$ with disjoint edges $e_{1}$ and $e_{6}$ of order 2 .

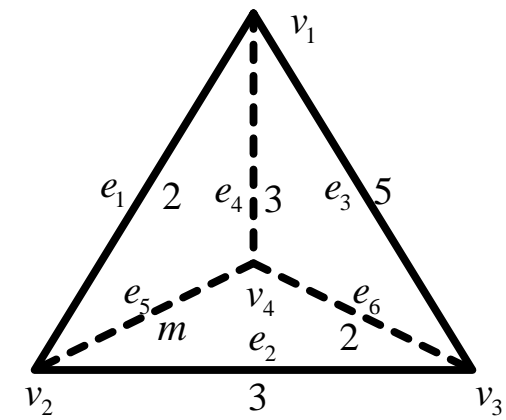

Figure 9: CHC Tetrahedron with Two vertices of order $(2,3,5)$ having disjoint edges of order 2 .

Then the possibilities of orders for $e_{5}$ are $m=2,3,4,5$.

For $m=2$, the order of $v_{2}$ becomes $(2,2,3)$ which is not possible by Theorem 4.1.

For $m=3$, the order of $v_{2}$ becomes $(2,3,3)$ which is not possible by Theorem 4.2 .

For $m=4$, the order of $v_{2}$ becomes $(2,3,4)$ which cannot be taken by assumption.

For $m=5$, it falls in case 1 . Hence, there is no such $T$ of this type.

Case 2.2: Two vertices of order $(2,3,5)$ with common edge

of order 2 .

Suppose the vertices $v_{1}$ and $v_{2}$ are of order $(2,3,5)$ with common edge $e_{1}$ of order 2 upto symmetry.

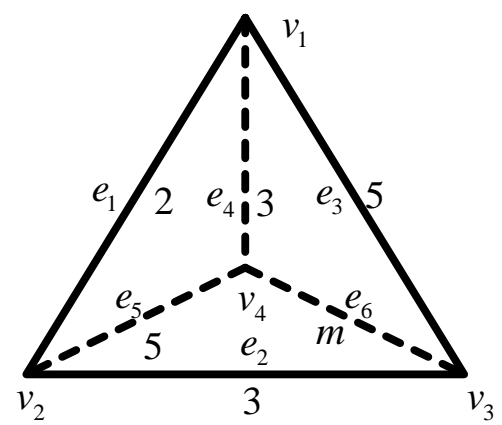

Figure 10: CHC Tetrahedron with Two vertices of order $(2,3,5)$ having common edge of order 2 .

Then the only possibility of order for $e_{6}$ is $m=2$ and this falls in case 1 . Hence, there is no such $T$ of this type.

Case 3: One vertex $v_{1}$ is of order $(2,3,5)$.

By corollary 4.4, the number of edges of order 2 at one vertex is exactly one and by corollary 4.5 , the edges of order 2 must be disjoint. Therefore if $v_{1}$ is of order $(2,3,5)$ with $e_{1}$ is of order 2 , then $e_{6}$ must be of order 2. Suppose the order of the edges $e_{2}$ and $e_{5}$ are $m_{1}$ and $m_{2}$ respectively.

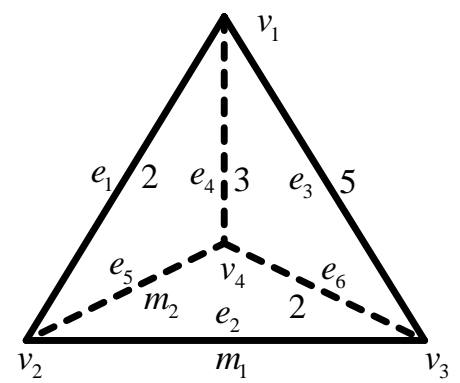

Figure 11: CHC Tetrahedron with one vertex of order $(2,3,5)$

To avoid symmetry, assume $m_{1} \leq m_{2}$. Therefore, $m_{1}=3$ and $m_{2}=3,4,5$ upto symmetry. For $m_{1}=3, m_{2}=3$, the order of $v_{2}$ becomes $(2,3,3)$ which is not possible by Theorem 4.2. For $m_{1}=3, m_{2}=4$, the order of $v_{2}$ becomes $(2,3,4)$ which cannot be taken by assumption. For $m_{1}=3, m_{2}=5$, it falls in case 1 . Hence, there is no such $T$ of this type.

From theorems 4.8 and 4.9, the total number of $\mathrm{CHC}$ tetrahedrons upto symmetry is: $2+1=3$. These are namely

$$
\begin{aligned}
& \text { CHCT-1 }=[2,3,4,3,4,2], \\
& \text { CHCT-2 }=[2,3,4,3,5,2], \\
& \text { CHCT-3 }=[2,3,5,3,5,2]
\end{aligned}
$$

These 3 tetrahedrons can be realized uniquely [10] in Hyperbolic space and these are nothing but the 3 coxeter Andreev's tetrahedrons found in theorems 3.20 and 3.21 in [1].

\section{CONCLUSIONS}

In this article, it is found that there are exactly $3 \mathrm{CHC}$ (compact hyperbolic coxeter) tetrahedrons upto symmetry in real projective space. These 3 tetrahedrons can be realized uniquely [10] in Hyperbolic space and these are nothing but the 3 coxeter Andreev's tetrahedrons found in theorems 3.20 and 3.21 in [1]. This research can be extended to other 
compact as well as non-compact hyperbolic polytopes in spaces of different dimensions. development of the template.

\section{REFERENCES}

[1] P. Kalita and B. Kalita, Properties of Coxeter Andreev's Tetrahedrons, IOSR Journal of Mathematics, Volume 9, Issue 6, pp 81-105, 2014.

[2] John G. Ratcliffe, Foundations of Hyperbolic Manifolds, (C1994 by Springer-Verlag, New York, Inc.

[3] Chris Godsil, Gordon Royle, Algebraic Graph Theory, Springer International Edition.

[4] Gil Kalai, Polytope Skeletons and Paths, (01997 by CRC Press LLC.

[5] Dipankar Mondal, Introduction to Reflection Groups, April 26, 2013, Triangle Group (Course Project).

[6] Projective Linear Group, http://en.wikipedia.org/wiki/Projective_linear_group, access in October, 2013.

[7] Hyperbolic Tetrahedron, http://mathworld.wolfram.com/Hyperbolic Tetrahedron.html, access in October, 2013.

[8] J. Mcleod, Hyperbolic Coxeter Pyramids, Advances in Pure Mathematics, Scientific Research, 2013, 3, 7882.

[9] Tetrahedron, Wikipedia, the free encyclopedia, access in October, 2013

[10] [10] Roland K. W. Roeder, Compact hyperbolic tetrahedra with non-obtuse dihedral angles, August 10, 2013, arxiv.org/pdf/math/0601148.

[11] Aleksandr Kolpakov, On extremal properties of hyperbolic coxeter polytopes and their reflection groups, Thesis No: 1766, e-publi.de, 2012.

[12] Anna Felikson, Pavel Tumaarkin, Coxeter polytopes with a unique pair of non intersecting facets, Journal of Combinatorial Theory, Series A 116 (2009) 875902.
[13] Pavel Tumarkin, Compact Hyperbolic Coxeter $n-$ polytopes with $n+3$ facets, The Electronic Journal of Combinatorics 14 (2007).

[14] R.K.W. Roeder, Constructing hyperbolic polyhedral using Newton's method, Experiment. Math. 16, 463492 (2007)

[15] Roland K.W. Roeder, John H. Hubbard and William D. Dunbar, Andreev's Theorem on Hyperbolic Polyhedra, Ann. Inst. Fourier, Grenoble 57, 3 (2007), 825-882.

[16] D. Cooper, D. Long and M. Thistlethwaite, Computing varieties of representations of hyperbolic 3-manifolds into $S L(4, \square), \quad$ Experiment. Math. 15, 291305 (2006).

[17] Yunhi Cho and Hyuk Kim. On the volume formula for hyperbolic tetrahedral. Discrete Comput. Geom., 22 (3): 347-366, 1999.

[18] Tomaz Pisanski, Milan Randic, Bridges between Geometry and Graph Theory, ISSN 1318-4865, Preprint Series, Vol. 36 (1998), 595.

[19] Raquel diaz, Non-convexity of the space of dihedral angles of hyperbolic polyhedra. C. R. Acad. Sci. Paris Ser. I Math., 325 (9):993-998, 1997.

[20] E. B. Vinberg, Geometry II, Encyclopedia of Maths, Sc 29. Springer 1993.

[21] E. B. Vinberg, Hyperbolic Reflection Groups, Uspekhi Mat. Nauk 40, 29-66 (1985)

[22] E. B. Vinberg, The absence of crystallographic groups of reflections in Lobachevskij spaces of large dimensions, Trans. Moscow Math. Soc. 47 (1985), 75-112.

[23] E. M. Andreev, On Convex Polyhedral of Finite Volume in Lobacevskii Space, Math. USSR Sbornik 10, 413-440 (1970). 